\title{
Characterization of Neisseria gonorrhoeae Strains Isolated from Patients with Conjunctivitis
}

\author{
Jorge Sosa ${ }^{+}$, Rafael Llanes, Wilder Rodríguez, Yainelys Gutiérrez, \\ Daymi Guzmán
}

\author{
Instituto de Medicina Tropical "Pedro Kourí”, Autopista Novia del Mediodía, km 6, Apdo Postal 601, \\ Marianao 13, Habana, Cuba
}

\begin{abstract}
The conjunctivitis produced by Neisseria gonorrhoeae is the less frequently reported clinical form of gonococcal infection. We aim to phenotypically characterize N. gonorrhoeae isolated from conjunctivae sites. A total of six cases of this disease were notified in the Camagüey province, Cuba. All the strains isolated were penicillin-producing, showed the serogroup WI and exhibited the same antimicrobial susceptibility pattern and plasmid profile (2.6-3.2-24.5). The results contribute to the characterization of $\mathrm{N}$. gonorrhoeae strains circulating in our environment.
\end{abstract}

Key words: Neisseria gonorrhoeae - conjunctivitis - characterization - Cuba

In 1943, the use of penicillin was introduced in gonorrhoea therapy and proved to be highly effective (Whittington \& Knapp 1988). A few years later some less susceptible strains appeared and, for some time, the use of 4.8 million units as single dose was effective to control the disease (Barnes \& Holmes 1984). Neisseria gonorrhoeae has shown a remarkable adaptation to the evolutionary pressure made for the treatment used in the last fifty years. Analysis of distribution and behaviour of antimicrobial resistant strains (Fekete 1993) have corroborated this. Few antimicrobial susceptibility studies using Minimal Inhibitory Concentration (MIC) methods and plasmid profiles have been carried out in our country, and it remains unknown which serogroups exist in our environment. The objective of the present study is to determine the susceptibility of six $N$. gonorrhoeae strains isolated from patients with conjunctivitis to penicillin, tetracycline, spec-tinomycin, ciprofloxacine and ceftriaxone, as well as the plasmid profile and serogroup.

A total of six strains were sent to the National Reference Laboratory of Pathogenic Neisseria at Pedro Kourí Institute in the Provincial Centre of

\footnotetext{
${ }^{+}$Corresponding author. Fax: $+537-246.05$. E-mail: sosa@ipk.sld.cu

Received 18 October 1999

Accepted 1 March 2000
}

Hygiene and Epidemiology in Camagüey. The strains were grown in a modified selective medium of Thayer-Martin, and by the Gram staining, oxidase test and superoxol test were identified as $N$. gonorrhoeae. Confirmation was performed using the traditional reference for utilization of carbohydrates and Phadebact Monoclonal GC OMNI test (Karo-Bio), which also allowed the determination of the serogroup. Beta-lactamase was detected using chromogenic cephalosporin test (Nitrocefin test, Oxoid). Antimicrobial susceptibility test on GC agar base medium supplemented with Isovitalex $1 \%$ (Becton \& Dickinson) was performed as previously described (NCCLS 1998 M7A3) and drugs recommended by the World Health Organization (penicillin, tetracycline, spectinomycin, ciprofloxacine and ceftriaxone) for the treatment of $N$. gonorrhoeae were tested. Plasmid DNA was extracted according to Birboim and Doly (1979).

The conjunctivitis produced by $N$. gonorrhoeae is a less frequent clinical form reported for gonococcal infection. A total of six cases of this disease in young adults were notified in Camagüey from May to June 1995. No relation was found between them nor the probable source of infection though clinical and epidemiological analysis were carried out. Cases presented severe ophthalmologic manifestations and low response to the antimicrobial therapy. Cultures of pharyngeal, rectal and genital specimens on selective media excluded other infections. When analyzing our results, we found that all strains were penicillin-producing $N$. gonorrhoeae (PPNG), showing high resistance 
levels to penicillin (MIC $\geq 16 \mathrm{mg} / \mathrm{ml}$ ) and tetracycline, although all strains were susceptible to the other antimicrobials. All strains showed identical plasmid profile (2.6-3.2-24.5 MDa) and belonged to WI serogroup.

Some authors have described gonococcal conjunctivitis outbreaks affecting different age groups but mainly those under 5 years of age. It has been suggested that unlike gonococcal infection at other location, a non-sexual mode of transmission may exist (Lewis et al. 1990).

The problem of high frequency of PPNG involvement in childhood conjunctivitis and adult gonococcal infection is aggravated by inadequate use of antibiotics (Odugbemi 1987). Most of our patients were first treated with penicillin and tetracycline. Studies developed in Cuba reported 68\% (56\% PPNG) and $84.6 \%$ of resistance to penicillin and tetracycline respectively in $N$. gonorrhoeae isolated from urogenital and conjunctivae specimens between 1995 and 1998 (Sosa 1999). Ebong et al. (1992) found that $68 \%$ of $N$. gonorrhoeae isolated from patients with conjunctivitis were resistant to penicillin (59.4\% PPNG) and most strains were susceptible to tetracycline. Interestingly our strains exhibited uniform value of MIC for the other antimicrobials (spectinomycin, ciprofloxacine and ceftriaxone). $\mathrm{MIC}_{90}$ of such drugs were $8 \mu \mathrm{g} / \mathrm{ml}$, $0.004 \mu \mathrm{g} / \mathrm{ml}$ and $0.008 \mu \mathrm{g} / \mathrm{ml}$, respectively. Many outbreaks caused by PPNG strains are associated with few phenotypes. Homogeneous data for antimicrobial susceptibility profile and serogroup were acquired. Dillon and Pauzé (1981) have described five strains of PPNG carrying this combination of plasmid (3.2-24.5 MDa) in Canada. Merianos et al. (1995) found WI (IA Outer Membrane Protein) serogroup as predominant in $N$. gonorrhoeae isolated from patients with conjunctivitis. In spite of such homogeneity, we believe other markers like auxotype, serovar and pulsed field pattern, could be used to support the possible relation of studied strains. The results contribute to the characterization of $N$. gonorrhoeae strains circulating in our environment.

\section{REFERENCES}

Barnes RC, Holmes KK 1984. Epidemiology of gonorrhoea: current perspectives. Epidemiol Rev 6: 1-31.

Birboim HC, Doly JA 1979. A rapid alkaline extraction procedure for screening recombinant plasmids. $\mathrm{Nucl}$ Acid Res 7: 1513-1523.

Dillon JR, Pauzé M 1981. Appearance in Canada of Neisseria gonorrhoeae strains with a 3.2 megadalton penicillin-producing plasmid and 24.5 megadalton transfer plasmid. Lancet ii: 700 .

Ebong EO, Utsalo SJ, Asindi AA, Archibong EI 1992. Penicillinase-producing Neisseria gonorrhoeae conjunctivitis on some Nigerian children. J Hyg Epidemiol Microbiol 36: 412-418.

Fekete T 1993. Antimicrobial susceptibility testing of Neisseria gonorrhoeae and implications for epidemiology and therapy. Clin Microbiol Rev 6: 22-23.

Lewis LS, Glauser TA, Joffe MD 1990. Gonococcal conjunctivitis in prepubertal children. Am J Dis Child 144: 546-548.

Merianos A, Condon RJ, Tapsall JW, Jayathissa S, Mulvey G, Lane JM, Patel MS, Rouse I 1995. Epidemic gonococcal conjunctivitis in central Australia. Med J Aust 162: 178-181.

NCCLS 1998. Methods for dilution antimicrobial susceptibility test for bacteria that grow aerobically. Document M7A3 (M100-S6).

Odugbemi T 1987. An open evaluative study of sublactam/ampicillin with or without probenecid in gonococcal infections in Lagos. Curr Therapy Res 41: 542-551.

Sosa J 1999. Caracterización Molecular de Cepas de Neisseria gonorrhoeae. Contribución a la Epidemiología, Msc Thesis, Instituto Pedro Kourí, Habana, $22 \mathrm{pp}$.

Whittington WL, Knapp JS 1988. Trends in resistance of Neisseria gonorrhoeae to antimicrobial agents in the United States. Sex Transm Dis 15: 202-210. 